\title{
Os debates sobre a natureza da posição de equilíbrio na Teoria Geral de Keynes
}

José Luís Oreiro ${ }^{1}$

Resumo: Este artigo tem por objetivo rediscutir a natureza da posição de equilíbrio com desemprego apresentada por Keynes na sua Teoria Geral à luz dos debates recentes em teoria macroeconômica, em particular, os debates entre James Tobin e Paul Davidson. Nesse contexto, iremos argumentar que (i) o modelo apresentado por Keynes na sua Teoria Geral não é um modelo com preços fixos, mas sim um modelo com preços flexíveis, o que significa dizer que não é válida a tese de que sua macroeconomia se caracteriza por uma suposta inversão da velocidade de ajuste entre preços e quantidades; (ii) o modelo de Keynes pressupõe rigidez da taxa nominal de salários ao longo de um dado período de produção, ao mesmo tempo em que admite flexibilidade (limitada) da taxa nominal de salários ao longo de uma seqüência de períodos; (iii) os efeitos dinâmicos da deflação dos salários nominais são, via de regra, desestabilizadores; e (iv) os efeitos dinâmicos da deflação dos salários nominais são, per se, incompatíveis com a definição de uma posição de equilíbrio com desemprego. Como corolário dessa argumentação segue-se que a essência da revolução Keynesiana deve ser buscada no campo da dinâmica econômica, ou seja, trata-se de uma demonstração da incapacidade do sistema de preços de produzir trajetórias não-explosivas para o sistema econômico.

Palavras-chave: Keynes; equilíbrio com desemprego; flexibilidade de preços; salários nominais.

\section{The debate on the nature of equilibrium in Keynes' General Theory}

Abstract: The nature of the equilibrium cum unemployment presented by Keynes in his General Theory is discussed in light of recent debates in macroeconomics. Special attention is paid to the views of James Tobin and Paul Davidson. As a 
corollary of this discussion, as we conclude, it follows that the essence of the Keynesian revolution is to be sought on the field of economic dynamics. In other words, it is about demonstrating the incapacity of the price system to create nonexplosive behavior in economic systems.

Key words: Keynes; equilibrium with unemployment; price flexibility; nominal wages.

JEL: E12; E24

\section{Introdução}

No prefácio à edição alemã da Teoria Geral do Emprego, do Juro e da Moeda [doravante TG], Keynes expõe a razão pela qual ele denomina a sua teoria de "geral", ao passo que a teoria clássica seria apenas um "caso particular". Para Keynes, a razão disso reside no fato de que a sua teoria estaria baseada num número menor de hipóteses restritivas do que a teoria clássica. Dessa forma, a sua teoria seria aplicável a um número maior de circunstâncias do que a teoria ortodoxa. Nas suas palavras (apud Davidson 1998:15): "This is one of the reasons which justify my calling my theory a General Theory. Since it is based on fewer restrictive assumptions than the orthodox theory, it is also more easily adopted to a large area of different circumstances."

Nesse contexto, a teoria clássica seria (Keynes 1936:3):

A special case only and not the general case (...) the characteristics of the special case assumed by the classical theory happen not to be those of the economic society in which we actually live, with the result that its teaching is misleading and disastrous if we attempt to apply it to the facts of experience.

Quais seriam as hipóteses menos restritivas que tornariam a Teoria de Keynes mais geral do que a Teoria Clássica? Sobre essa questão existiram grandes controvérsias ao longo da história do desenvolvimento da teoria macroeconômica. Uma leitura possível da TG de Keynes é que a mesma não passaria de um caso particular da teoria clássica, ou seja, o caso em que preços e/ou salários nominais são rígidos. Embora a rigidez nominal possa ser considerada um "fato da vida", do ponto de vista teórico trata-se de uma hipótese mais restritiva do que a hipótese de flexibilidade de preços e salários nominais, uma vez que carece de fundamentos microeconômicos adequados, (cf. Amadeo 1992:140). Essa linha de interpretação da TG foi pioneiramente apresentada por Modigliani (1944), permanecendo até os dias de hoje como a "leitura 
dominante" da obra de Keynes, notadamente nos centros de pós-graduação em economia com perfil mais próximo ao mainstream. ${ }^{2}$ Nesse contexto, a contribuição de Keynes ao pensamento econômico limitarse-ia à constatação de que, face às rigidezes nominais existentes no sistema econômico, caberia à política econômica um papel importante na regulação das economias de mercado. No entanto, ao nível da teoria pura, a teoria de Keynes não seria uma alternativa real à teoria clássica, mas apenas um caso particular da mesma (cf. Carvalho 1992:8). Essa postura fica bem clara na seguinte citação de Solow e Hahn (1986:5):

The dominant reading of the General Theory - though not the only one - seems nevertheless to have ticked Keynes as the theorist of stickyprice macroeconomics. His suggestion (...) that wage and price flexibility, if it were possible, might be a poor or even self-defeating way to achieve equilibrium at full employment, seems more or less to have disappeared from professional consciousness.

Essa interpretação da TG não é, contudo, unânime entre os macroeconomistas. Mesmo economistas do mainstream da ciência econômica como James Tobin argumentam que a TG não é um simples caso particular da Teoria Clássica. Segundo Tobin (1993), a generalidade da TG de Keynes se assenta no fato de que a mesma pressupõe que a velocidade de ajuste de preços e salários a divergências entre demanda e oferta se situa entre zero e infinito, ao passo que o "modelo clássico" pressupõe ajuste instantâneo de preços e salários. Em outras palavras, os resultados e as prescrições da teoria clássica exigem um tipo bastante particular de flexibilidade de preços, qual seja: a flexibilidade instantânea. Isso significa que face a um choque de demanda ou de oferta os preços devem "saltar" instantaneamente para os seus valores de equilíbrio; do contrário, o ajuste incompleto dos preços nominais ensejaria a um ajuste nas quantidades produzidas e no nível de emprego, dando origem a uma dinâmica tipicamente Keynesiana. Em carta enviada a Paul Davidson em 5 de maio de 1993, Tobin deixa bem clara a sua posição (apud Davidson 1998:22):

I regard 'perfect flexibility' as a condition that never exists, never can exist. As I define it, it means that never for any finite period of time, however, short, do supplies and demands at existing prices diverge. This means that any shocks to supply and demand are absorbed by 'jumps' in prices, so that there is no period of real time during which

2 No Brasil a única exceção a essa regra entre os centros ortodoxos era, até meados da década de 1990, o Departamento de Economia da PUC-RJ em função da presença de um dos maiores especialistas em economia keynesiana no país, o professor Edward Amadeo. 
prices are adjusting, but have not yet fully adjusted, to the shock.

Na visão de Tobin, a flexibilidade em tempo real - ou seja, não-instantânea - de preços e salários nominais não seria capaz, em geral, de conduzir a economia a uma posição de equilíbrio com pleno-emprego, caso a mesma tenha se afastado dessa posição em função da ocorrência de um choque de demanda e/ou de oferta. Isso porque a deflação de preços e/ ou salários nominais provocaria uma contração da demanda agregada, a qual atuaria no sentido de amplificar a queda do nível de produção e de emprego, acelerando assim um processo deflacionário. Deve-se observar, no entanto, que esse processo não só não conduz a economia a uma posição de equilíbrio com pleno-emprego, como também não conduz a nenhum tipo de equilíbrio, ou seja, não resultaria num equilíbrio com desemprego. Para tanto, seria necessário que a deflação de preços e salários nominais não tivesse nenhum tipo de efeito (nem positivo e nem negativo) sobre a demanda agregada, o que seria per se uma hipótese tão ou mais restritiva do que a hipótese de flexibilidade instantânea de preços e salários nominais. Dessa forma, a essência da TG de Keynes deve ser vista como uma demonstração da incapacidade do sistema de preços de garantir a manutenção do pleno-emprego ao longo do tempo, ao invés de ser entendida como uma demonstração da existência de um equilíbrio com desemprego. Nas palavras de Tobin (1975:195-96):

\begin{abstract}
The real issue is not the existence of a long-run static equilibrium with unemployment, but the possibility of protracted unemployment which the natural adjustments of a market economy remedy very slowly if at all. So what if, within the recherché rules of contest, Keynes failed to establish an 'underemployment equilibrium?' The phenomena he described are better regarded as disequilibrium dynamics (...) The Great Depression is the Great Depression, the notorious 'Treasury View' is still ridiculous, whether mass unemployment is a feature of an equilibrium or of a prolonged disequilibrium.
\end{abstract}

Essa interpretação de Tobin não tem sido aceita, contudo, pelos economistas pós-keynesianos e, em particular, por Paul Davidson, para quem a leitura de Tobin da TG de Keynes seria uma simples reformulação da interpretação feita por Leijonhufvud (1968). Segundo Davidson, o aspecto essencial do modelo apresentado por Keynes na TG seria a reversão da velocidade relativa de ajuste entre preços e quantidades suposta por Alfred Marshall. Se, para Marshall, os preços são a variável que se ajusta primeiro a um choque de demanda, para Keynes seriam as quantidades que se ajustariam primeiro a esse tipo de choque. Essa mesma interpretação é endossada por Friedman nos seguintes termos (apud Davidson 1997:255): 
It takes time for output to adjust but no time for prices to do so. This assumption [of Marshall] has no effect on the final equilibrium position, but it is vital for the path to equilibrium (...) Keynes (...) deviated from Marshall (...) in reversing the roles assigned to prices and quantities (...) Keynes's assumption about the relative speeds of adjustment of price and quantities is still the key difference in approach and analysis between those economists who regard themselves as Keynesians and those who do not.

A crítica de Davidson à interpretação de Tobin vai no sentido de que a mesma atribui a uma imperfeição localizada no lado da oferta da economia a causa do desemprego involuntário da força de trabalho (cf. Davidson 1998:18). No entanto, argumenta Davidson, Keynes teria reiteradas vezes afirmado que (i) a sua teoria seria aplicável a economias com graus variados de concorrência (o que inclui a concorrência perfeita e, portanto, uma situação de perfeita flexibilidade de preços); (ii) o fator que limita a criação de emprego no mundo real é a demanda efetiva, e não as condições de oferta da economia (Davidson 1998:18).

Isso posto, o presente artigo tem por objetivo rediscutir a natureza da posição de equilíbrio com desemprego apresentada por Keynes na sua Teoria Geral. Nesse contexto, iremos argumentar que (i) o modelo apresentado por Keynes na sua Teoria Geral não é um modelo com preços fixos, como supõe a maior parte dos economistas ligados à macroeconomia convencional, mas é um modelo com preços flexíveis, o que significa dizer que não é válida a tese de que a macroeconomia de Keynes se caracteriza por uma suposta inversão da velocidade de ajuste entre preços e quantidades; (ii) o modelo de Keynes pressupõe rigidez da taxa nominal de salários ao longo de um dado período de produção, ao mesmo tempo em que admite flexibilidade da taxa nominal de salários ao longo de uma seqüência de períodos; (iii) os efeitos dinâmicos da deflação dos salários nominais são, via de regra, desestabilizadores, ou seja, a redução de salários nominais tende, em geral, a produzir um processo cumulativo de contração da demanda agregada e, conseqüentemente, do nível de emprego; e (iv) os efeitos dinâmicos da deflação dos salários nominais são, per se, incompatíveis com a definição de uma posição de equilíbrio com desemprego, uma vez que o equilíbrio é visto na Teoria Geral como uma posição de repouso do sistema econômico.

Como corolário dessa argumentação, segue-se que a essência da revolução Keynesiana deve ser buscada no campo da dinâmica econômica, ou seja, trata-se de uma demonstração da incapacidade do sistema de preços de produzir trajetórias não-explosivas para o sistema econômico, demandando assim a intervenção governamental como forma de estabilizar a economia. O conceito de "equilíbrio com desemprego" é, nesse contexto, logicamente incorreto e teoricamente desnecessário. $\mathrm{O}$ que importa para a economia Keynesiana é a análise das trajetórias que 
a economia descreve fora do equilíbrio. Além disso, devemos destacar que a conclusão final de nossa argumentação é que a leitura de Tobin da obra de Keynes não é interpretação totalmente equivocada da mesma, como afirma Davidson, mas uma leitura que apresenta de maneira consistente a essência da contribuição de Keynes para a teoria econômica.

Para tanto, o presente artigo está organizado em cinco seções, incluindo a presente introdução. Na seção seguinte, iremos apresentar um modelo formal da teoria do emprego de Keynes com base em Amadeo (1992) com o objetivo de explicitar os pressupostos teóricos da mesma e mostrar que essa teoria não está baseada na hipótese de preços fixos. Em seguida, iremos apresentar os argumentos desenvolvidos por Keynes no capítulo 19 da TG com o intuito de mostrar que a deflação de salários nominais pode gerar uma contração - ao invés de uma expansão - do nível de demanda efetiva, de forma a resultar numa redução (ao invés de uma expansão) do nível de renda e de emprego. Na seção posterior, iremos discutir o conceito de equilíbrio apresentado por Keynes na TG de forma a avaliar em que medida é correto afirmar que a TG estabelece as condições formais de existência de uma posição de equilíbrio com desemprego. A seção final sumariza as conclusões obtidas ao longo do artigo.

\section{A teoria do emprego de Keynes e a velocidade relativa de ajuste entre preços e quantidades}

Na TG, Keynes considera uma economia ancorada no curto-prazo Marshalliano, ou seja, "o menor intervalo de tempo após o qual a firma está livre para rever as suas decisões sobre o volume de emprego a oferecer" (Keynes 1936:47). Em outras palavras, no curto-prazo, o nível de emprego e, conseqüentemente, o nível de produção e utilização da capacidade produtiva, são variáveis sobre as quais as firmas podem tomar decisões. Dessa forma, o curto-prazo na TG pode ser identificado com o período de produção, ou seja, com o intervalo de tempo compreendido entre o momento no qual a firma decide o número de trabalhadores a serem contratados - e, portanto, o volume de bens a serem produzidos - e a venda da sua produção acabada no mercado.

Como a produção é um processo que demanda tempo, ${ }^{3}$ segue-se que a decisão de produção e de contratação de trabalhadores é tomada com base nas expectativas que as firmas formam no início do período de produção a respeito da receita que serão capazes de obter com a venda de sua produção no mercado. Sendo assim, a decisão de produção é

3 Nas palavras de Asimakopulos (1991:40): "Production decisions made by Keynes's competitive firms must be based on expectations of prices, because of the gap between the time when these decisions are made, and the time when the resulting output is ready for sale." 
informada por expectativas de curto-prazo, definidas por Keynes (1936: 46) como: "The price which a manufacturer can expect to get for his 'finished' output at the time when he commits himself to starting the process which will produce it; output being 'finished' (from the point of view of the manufacturer) when it is ready to be sold to a second party."

Tal como se observa na citação acima de Keynes, as expectativas de curto-prazo referem-se ao preço pelo qual a firma espera vender a sua produção. Nada é dito a respeito das quantidades que ela espera ser capaz de vender no mercado. Daqui se conclui que a estrutura de mercado suposta por Keynes na TG é a concorrência perfeita, uma vez que nessa estrutura de concorrência a firma é tomadora de preços e fixadora de quantidades, não cabendo às condições de demanda nenhum papel direto $^{4}$ na determinação da quantidade produzida. ${ }^{5}$

Isso posto, no capítulo 3 da TG Keynes define a demanda agregada, D, como sendo a receita que os empresários esperam receber da venda de sua produção acabada no mercado ${ }^{6}$. Temos, então, que:

$$
\text { (1) } D=E[p] X
$$

Onde: $E[p]$ é o preço unitário que as firmas esperam receber no momento da venda de sua produção no mercado; $X$ é a quantidade produzida.

A quantidade produzida, $X$, é constituída por bens de consumo e bens de capital. Supondo que o preço relativo dos bens de consumo em termos dos bens de capital é constante e igual a um, então a quantidade produzida pode ser expressa simplesmente como a soma entre a quantidade produzida de bens de consumo $\left(X_{c}\right)$ e a quantidade produzida de bens de capital $\left(X_{l}\right)$. Sendo assim, a demanda agregada pode ser expressa como:

$$
\text { (2) } D=E[p] X_{c}+E[p] X_{k}
$$

$\mathrm{Na}$ equação (2), o primeiro termo do lado direito representa o valor esperado dos gastos nominais de consumo, ao passo que o segundo termo representa o valor esperado dos gastos nominais de investimento.

4 As condições de demanda têm, no entanto, um papel indireto. Uma elevação da demanda agregada irá resultar num aumento do nível de preços e, dadas as curvas de custo marginal de cada empresa, irá induzir um aumento do nível de produção e de emprego. Contudo, cada empresa dessa economia acredita ser capaz de vender toda a quantidade por ela produzida aos preços de mercado, de maneira que a demanda não impõe um limite à expansão da produção a nível da firma individual.

5 Uma outra prova de que a teoria do emprego de Keynes está construída sob a hipótese de concorrência perfeita é o fato de que no capítulo 2 da TG Keynes é bastante explícita na aceitação do "primeiro postulado" da Teoria Clássica do emprego, segundo o qual o salário real é igual à produtividade marginal do trabalho (cf. Keynes 1936:5-6).

6 O modelo aqui apresentado segue Amadeo (1992). 
Iremos supor que a quantidade produzida de bens de capital $\left(X_{k}\right)$ é uma constante exógena, uma vez que a produção de bens de capital é feita sob encomenda e, portanto, dependente das decisões de investimento dos empresários. Essas decisões de investimento, por sua vez, dependem do estado de expectativas de longo-prazo, ou seja, da receita que os empresários esperam obter do equipamento de capital por eles adquirido ao longo do período de vida útil desse mesmo equipamento (cf. Keynes 1936:46-7). Ao contrário das expectativas de curto-prazo, as expectativas de longo-prazo não são revistas a intervalos curtos, de maneira que as mesmas podem ser consideradas como dadas do ponto de vista da decisão de produção e de emprego (cf. Asimakopulos 1991: 39). ${ }^{7}$

Consideremos agora que os produtores de bens de consumo conhecem a propensão marginal a consumir da sociedade - a qual iremos designar por $j$ - mas não conhecem a renda nominal agregada. Dessa forma, o valor esperado dos gastos nominais de consumo, $E(C)$, pode ser expresso por:

$$
\text { (3) } E(C)=E(p) X_{c}=j\left[E(p)\left(X_{c}+X_{j}\right)\right]=j E[p] X
$$

Substituindo (3) em (2), obtemos:

$$
\text { (4) } D(N)=j E[p] A(N) N+E[p] X_{k}
$$

Onde: $N$ é o nível de emprego; $A(N)=\frac{X}{N}$ é a produtividade média do trabalho na economia como um todo ${ }^{8}$.

Keynes define a oferta agregada, $(Z)$, como o preço de oferta de um dado volume de produção (cf. Keynes 1936:24-5). Mais concretamente, podemos definir a oferta agregada como a receita requerida para cobrir os custos associados a um determinado volume de produção, o que inclui a taxa normal de retorno sobre o capital. Como em concorrência perfeita o lucro econômico é igual a zero, segue-se que a receita requerida será igual ao produto entre o custo marginal de produção e a quantidade produzida.

Na construção da função de oferta agregada, iremos supor que as firmas tomam o salário nominal como um dado (cf. Asimakopulos 1991: 45). Isso porque, ao se iniciar o período de produção, as firmas e os trabalhadores negociam a taxa de salário nominal que irá prevalecer ao

7 Isso não quer dizer, no entanto, que essas expectativas não possam ser alteradas ao longo de uma sucessão de curtos-prazos como decorrência de erros de previsão a respeito da receita obtida com o equipamento de capital.

8 Fazendo o suposto tradicional de rendimentos marginais decrescentes do fator variável, seguese que a produtividade média do trabalho será uma função decrescente do nível de emprego, N. 
longo do mesmo. Isso não quer dizer que os salários nominais sejam fixos. A taxa nominal de salários pode ser alterada ao longo de uma sequência de curtos-prazos como decorrência da situação prevalecente no mercado de trabalho. Para a teoria do emprego de Keynes é necessário apenas que os salários nominais não mudem ao longo de um dado período de produção.

O custo marginal de produção, no caso em que o trabalho é o único insumo variável utilizado no processo produtivo, é dado por $\frac{W}{F^{\prime}(N)}$, onde $W$ é a taxa nominal de salários e $F^{\prime}(N)$ é a produtividade marginal do trabalho. Dessa forma, a oferta agregada pode ser expressa por: ${ }^{9}$

(5) $Z(N)=\frac{W}{F^{\prime}(N)} X=\frac{W}{F^{\prime}(N)} \frac{X}{N} N=\frac{W}{F^{\prime}(N)} A(N) N$

O nível de emprego é determinado no ponto em que a demanda e a oferta agregada são iguais, uma vez que, nesse ponto, as firmas estarão maximizando o seu lucro esperado (cf. Dutt 1992: 209). Após os algebrismos necessários, obtemos a seguinte expressão:

(6) $X^{*}=A\left(N^{*}\right) N^{*}=\frac{E[p] X_{k}}{\left[\frac{W}{F^{\prime}\left(N^{*}\right)}-j E[p]\right]}$

Onde: $X^{*}$ é o nível de produção que maximiza os lucros esperados das firmas e $N^{*}$ é o nível de emprego que maximiza os lucros esperados das firmas.

A equação (6) mostra que o nível de produção que maximiza os lucros esperados das firmas depende das suas expectativas a respeito do preço pelo qual serão capazes de vender a sua produção no mercado, do volume físico de produção da indústria produtora de bens de capital, da propensão marginal a consumir e da taxa nominal de salários.

Uma vez que as firmas tenham decidido o quanto produzir com base na equação (6), a quantidade produzida não mais poderá ser alterada ao longo do período de produção. Isso significa que mudanças não-previstas nas condições de demanda da economia (os assim chamados "choques de demanda agregada") irão se refletir integralmente em variações nos preços dos produtos, e não nas quantidades produzidas, gerando assim "lucros" ou "prejuízos" extraordinários (windfall profits or losses), os quais poderão dar ensejo a mudanças nas expectativas de curto-prazo dos empresários no próximo período de produção. Em outros termos, choques de demanda geram primeiramente uma mudança nos pre-

9 Uma derivação semelhente pode ser obtida em Chick (1993:73). 
ços dos produtos e, como conseqüência dessas mudanças de preços, ocorrem alterações nas quantidades produzidas ao longo de uma seqüência de curtos-prazos.

Para que se possa entender o funcionamento desse mecanismo de ajuste, defina-se $G_{t}^{*}$ como a demanda realizada no período $t$. A demanda realizada é a soma dos gastos realizados de consumo $\left(j \cdot p_{t} \cdot X_{t}^{*}\right)$ com os gastos realizados de investimento $\left(p_{t} X_{t}^{k}\right)^{10}$. Defina-se o lucro ou prejuíso extraordinário como a diferença entre a demanda realizada e as expectativas das firmas a respeito da receita decorrente da venda de seus produtos, ou seja, a demanda agregada no sentido definido por Keynes (cf. Amadeo 1992:154). Dessa forma, temos que:

$$
\text { (7) } G_{t}^{*}-D\left(N_{t}^{*}\right)=\left[p_{t}-E\left[p_{t}\right]\right]\left(j \cdot X_{t}^{*}+X_{t}^{k}\right)
$$

Com base na equação (7), podemos constatar que se a demanda realizada for maior do que as expectativas de receita das firmas então o preço pelo qual as empresas conseguiram efetivamente vender a sua produção no mercado ao final do período é maior do que a expectativa que elas tinham inicialmente a respeito do mesmo. Esse erro de previsão irá resultar num lucro acima do esperado pelas empresas, sinalizando, por um lado, a necessidade de se rever as expectativas quanto ao preço de venda e, por outro, a possibilidade de aumentar o nível de produção e de emprego de forma lucrativa.

Pela equação (6), sabemos que o nível de produção e de emprego decidido pelas firmas no início de cada período de produção é uma função crescente do preço esperado. Sendo assim, um aumento imprevisto da demanda no período $t$, que tenha resultado num lucro extraordinário nesse mesmo período como decorrência do fato de $p_{t}>E\left[p_{t}\right]$, irá induzir um aumento do preço esperado de venda para o período de produção e, portanto, irá resultar num aumento do nível de produção e de emprego no período $t+1$. Daqui se segue que, face a um "choque de demanda", os preços se ajustam antes das quantidades no modelo desenvolvido por Keynes na TG. Não é, portanto, verdade que a teoria do emprego de Keynes se baseie numa reversão das velocidades relativas de ajuste entre preços e quantidades com respeito à suposição tradicional feita por Alfred Marshall. No que se refere a esse aspecto, a interpretação que Tobin faz da TG é incorreta.

10 Onde: $p_{t}$ é o nível efetivo de preços no período $t$ e $X_{t}^{*}$ é o nível de produção decidido pelas firmas no início do período $t$. 


\section{Salários flexíveis, emprego e demanda agregada: revisitando o capitulo 19 da Teorza Geral}

Uma outra fonte de controvérsias no que se refere à interpretação da TG é o papel que a hipótese de "rigidez" de salários nominais desempenha no sistema de Keynes. Vimos na seção anterior que a teoria do emprego de Keynes realmente pressupõe que os salários nominais são dados ao longo do período de produção. Além disso, Keynes passa os primeiros 18 capítulos da TG supondo que os salários nominais não se alteram. Apenas no capítulo 19 é que Keynes analisa os efeitos de mudanças da taxa de salário nominal sobre a demanda realizada e, portanto, sobre o nível de produção e de emprego.

De acordo com o saber convencional reinante entre os economistas acadêmicos, a teoria de Keynes realmente estaria baseada na hipótese de rigidez de salários nominais ${ }^{11}$. Isso porque, sem essa hipótese, seria impossível demonstrar a existência de um equilíbrio com desemprego involuntário da força de trabalho. Se os salários forem flexíveis à queda, então a existência de desemprego involuntário irá forçar os salários nominais para baixo, produzindo assim uma queda do nível geral de preços. A deflação de preços, por sua vez, irá resultar num aumento do valor real da riqueza dos agentes econômicos num contexto em que o estoque de outside money (papel-moeda em poder do público) é constante. Em função do aumento do valor real do seu estoque de riqueza, os agentes econômicos irão aumentar os seus gastos de consumo, gerando assim uma expansão da demanda realizada e, dessa forma, do nível de produção e de emprego. Trata-se do assim chamado efeito liquidez-real (também conhecido como efeito Pigou-Patinkin), cuja operação resulta num processo mais ou menos rápido de convergência em direção a uma posição de equilíbrio com pleno-emprego (cf. Patinkin 1948:259-60).

Essa interpretação do papel da hipótese de rigidez de salários nominais na TG de Keynes tem sido negada por vários economistas, alguns dos quais pertencentes também ao mainstream da ciência econômica. Por exemplo, Solow e Hahn (1986:3) afirmam que "Keynes did not think that nominal-wage rigidity was the sole or main cause of unemployment. Indeed (...) he was at pains to argue that wage cuts were not the proper cure for unemployment, and might be no cure at all." Um outro exemplo é Tobin (1975:195), que afirma: "Keynes tried to make a double argument about wage reductions and employment. One was that wage rates were very slow to decline in face of excess supply. The other was

11 A esse respeito, vejamos o que diz Modigliani (1944:223): "The liquidity preference theory is not necessary to explain underemployment equilibrium; it is sufficient only in a limiting case: the 'Keynesian case.' In the general case it is neither necessary nor sufficient; it can explain this phenomenon only with the additional assumption of rigid wages." 
that, even if they declined faster, employment would not - in depression circumstances - increase."

Para que possamos fazer uma avaliação correta do papel da flexibilidade de salários no sistema de Keynes, devemos ter em mente que existem duas questões teoricamente distintas sendo debatidas. A primeira refere-se à relação entre o nível da taxa nominal de salários e a demanda realizada. Mais especificamente, trata-se de averiguar se existe uma relação inversa entre o nível de salários nominais e a demanda realizada tal como é suposto pelo efeito liquidez-real. Se a resposta a essa pergunta for afirmativa, então a flexibilidade de salários irá conduzir a economia, no longo-prazo, a uma posição de equilíbrio com pleno-emprego, de forma que a ocorrência de desemprego involuntário da força de trabalho só pode resultar da "lentidão" com a qual os salários nominais se ajustam. Caso contrário, o desemprego involuntário de Keynes não depende de hipóteses específicas a respeito do comportamento da taxa nominal de salários. A segunda questão é averiguar se é possível a ocorrência de um "equilíbrio", entendido como posição de repouso do sistema econômico, num contexto em que os salários nominais são flexíveis.

Uma resposta negativa à primeira pergunta não implica necessariamente numa resposta afirmativa à segunda. Em outras palavras, é logicamente possível se afirmar simultaneamente que a flexibilidade dos salários nominais não garante a convergência à posição de equilíbrio com pleno-emprego, mas também que essa flexibilidade impede que o sistema econômico alcance uma posição de equilíbrio com desemprego da força de trabalho. Nesse caso, a flexibilidade de salários resultaria numa situação de "desequilíbrio com desemprego." Nesta seção iremos tratar da primeira questão, deixando a segunda questão para a seção seguinte.

No capítulo 19 da TG, Keynes afirma que a redução dos salários nominais teria os seguintes efeitos sobre a demanda realizada numa economia sem relações com o exterior:

1. Haveria uma redistribuição de renda dos salários para os lucros, uma vez que a redução dos salários seria maior do que a dos preços ${ }^{12}$. Supondo que a propensão a consumir a partir dos lucros é maior do que a propensão a consumir a partir dos salários, isso terá um efeito depressivo sobre a demanda realizada.

2. Caso se espere que a redução dos salários nominais será once-andfor-all, isto é, relativamente aos salários nominais no futuro, então isso será favorável ao investimento, uma vez que, para um mesmo estado de 
expectativas de longo-período, a eficiência marginal do capital para os investimentos feitos hoje será maior do que a dos investimentos realizados no futuro ${ }^{13}$. Entretanto, se isso provocar uma expectativa de novas reduções de salários no futuro, segue-se que poderá se obter uma maior taxa de retorno para os investimentos em capital fixo se os mesmos forem adiados. Nesse caso, a deflação é prejudicial aos investimentos e à demanda realizada.

3. A deflação provocará uma redução da renda nominal, diminuindo a demanda nominal de moeda para fins transacionais. Isso provocará uma redução da taxa de juros, o que estimulará o investimento. Este é famoso Efeito Keynes.

4. Como a redução de salários é sempre vantajosa para um empresário individual, uma vez que ela reduz os seus custos de produção, uma deflação generalizada pode produzir um clima de otimismo entre os empresários, ou seja, pode aumentar o seu grau de confiança com respeito ao futuro. Isso teria o efeito de reduzir a preferência pela liquidez, diminuindo as taxas de juros e estimulando o investimento.

5. Por fim, a deflação aumenta o peso real das dívidas, podendo provocar uma onda de insolvências. Fora isso, um maior valor real para as dívidas irá reduzir a propensão a consumir dos devedores. Se esta for maior do que a propensão a consumir dos credores, segue-se que o efeito será depressivo sobre a demanda agregada.

Deve-se observar que, dos cinco argumentos apresentados, três apontam para a existência de um efeito negativo da deflação sobre o dispêndio agregado e dois para a existência de um efeito positivo. Dessa forma, numa análise puramente teórica, uma redução de salários nominais tem efeito ambíguo sobre a demanda realizada, ou seja, não é necessariamente verdade que a relação entre o nível de salários nominais e a demanda realizada seja inversa (cf. Amadeo 1989:147). Nesse contexto, é perfeitamente possível que a redução de salários nominais resultante da ocorrência de desemprego involuntário da força de trabalho faça com que o nível de produção e de emprego se reduza ainda mais, amplificando assim o tamanho do desemprego. Portanto, não é verdade que a flexibilidade de salários nominais seja condição suficiente para garantir a convergência a uma posição de equilíbrio com pleno-emprego da força de trabalho. Muito pelo contrário! Essa flexibilidade pode terminar por gerar um círculo vicioso de deflação dos salários nominais-queda da demanda realizada-contração do nível de emprego-

13 Isso se constata da seguinte forma: a eficiência marginal do capital é definida como a taxa de desconto que equaliza o preço de oferta dos bens de capital recentemente produzidos com as receitas que se esperam obter de sua utilização. Se a redução dos salários implicar numa diminuição do preço de oferta dos bens de capital, então a taxa de desconto deve aumentar para manter a referida igualdade. 
nova queda dos salários nominais ${ }^{14}$. Sendo assim, podemos afirmar que a existência de desemprego involuntário da força de trabalho não depende da hipótese feita a respeito do comportamento da taxa nominal de salários.

Uma última observação é necessária sobre os argumentos do capítulo 19 da TG. Alguns autores afirmam que a flexibilidade de salários nominais só não levará a economia a uma posição de equilíbrio com plenoemprego caso a economia esteja operando sob condições de armadilha da liquidez. Um exemplo disso é Friedman (apud Leijonhufvud 1981: 190) que afirma que: "Time and again when Keynes must face up to precisely what is that prevents a full-employment equilibrium, his final line of defense is absolute liquidity preference."

No que se refere a esse ponto, devemos observar que em nenhum momento ao longo do capítulo 19 da $\mathrm{TG}$, Keynes faz referência à armadilha da liquidez para demonstrar que a flexibilidade de salários nominais não conduz à posição de equilíbrio com pleno-emprego. A dita armadilha não se acha explicitada entre os efeitos de uma deflação de salários sobre a demanda agregada. Antes, pelo contrário, a inclusão do efeito Keynes mostra que a redução de salários e preços tem efeito sobre a taxa de juros, ao contrário do que ocorreria na referida situação.

Além disso, no momento em que Keynes afirma que a deflação salarial, como forma de promover o emprego, estaria sujeita às mesmas limitações da política monetária (Keynes 1936:266), seu argumento subseqüente torna claro que o que ele tinha em mente não era a armadilha da liquidez, mas o efeito que variações de diferentes magnitudes da oferta de moeda poderiam ter sobre o estado de preferência pela liquidez, ao aumentar ou reduzir a confiança dos empresários sobre o futuro. Nas suas palavras (Keynes 1936:267):

It follows that wage-reductions, as a method of securing full employment, are also subject to the same limitations as the method of increasing the quantity of money. The same reasons as those mentioned above, which limit the efficacy of increases in the quantity of money as means to increase investment to the optimum figure, apply mutatis mutandis to wage reductions. Just as a moderate increase in the quantity of money may exert an inadequate influence over the longterm rate of interest, whilst an immoderate increase may offset its

14 É precisamente em função da possibilidade lógica de ocorrência desse ciclo vicioso que Keynes acreditava que a estabilidade da taxa nominal de salários era uma condição necessária para a estabilidade das economias de mercado. Nas suas palavras: "If competition between unemployed workers always led to a very great reduction of the money-wage rate, there would be a violent instability in the price-level. Moreover, there might be no position of stable equilibrium except in conditions of full-employment; since the wage unit might have to fall without limit until it reached a point where the effect of the abundance of money in terms of the wage-unit on the rate of interest was sufficient to restore a level of full-employment" (1936:253). 
other advantages by its disturbing effect on confidence; so a moderate reduction in money-wages may prove inadequate.

\title{
3. Equilíbrio com desemprego ou desemprego de desequilíbrio?
}

Na seção anterior vimos que os argumentos apresentados por Keynes no capítulo 19 da TG apontam para a idéia de que a flexibilidade de salários nominais não é capaz de garantir a convergência das economias de mercado à posição de equilíbrio com pleno-emprego. Sobre esse aspecto há uma notável convergência de opiniões entre Keynesianosneoclássicos - como, por exemplo, Tobin - e pós-keynesianos - como, por exemplo, Paul Davidson. Com efeito, Tobin (1997:20) afirma que:

\begin{abstract}
Once excess-supply-reducing price adjustments are acknowledged to take real time, the destabilizing effects of the process - falling prices rather than fallen prices - become important. In the context of the great macroeconomic debate, expectations of declining money-wages and prices are bad for real aggregate demand. They raise real interest rates. In comparison, the real balance effect will be at best weak and at worst wrong in sign... The above argument concerns the possible instability of the classical full-employment equilibrium, and the time required for adjustments even if the process is stable.
\end{abstract}

Essa afirmação não é muito diferente da opinião de Davidson (1997: 255) sobre o assunto, para quem "Keynes insisted that as a logical conclusion of his analysis no market price mechanism (including completely flexible prices) exists that ensures a self-righting fullemployment equilibrium in a monetary economy."

Sendo assim, por que razão Davidson (1998:17) insiste em afirmar que "Tobin's interpretation of Keynes is in expositional and logical conflict with Keynes's own writings on what is essential to and the substance of the general theory of employment?"

Uma resposta possível a essa pergunta consiste no fato de que a interpretação de Tobin da TG de Keynes se apóia na hipótese de reversão da velocidade relativa de ajuste entre preços e quantidades. De fato, Tobin afirma que "ele [Keynes] acreditava que os salários e os preços são flexíveis, mas de forma imperfeita" (1997:19, tradução nossa). Conforme foi mostrado na seção 2 deste artigo, a teoria do emprego de Keynes assume preços instantaneamente flexíveis, ou seja, flexíveis ao longo de um dado período de produção. No entanto, o mesmo não ocorre com os salários nominais. Estes só podem variar entre períodos, mas não em um dado período de produção. Sendo assim, a interpretação de Tobin 
não é (plenamente) compatível com a "essência" e a "substância" da teoria do emprego de Keynes (cf. Amadeo 1989:145).

No entanto, existe um aspecto da argumentação de Tobin que Davidson não leva em consideração, a saber: a incompatibilidade entre a hipótese de salários flexíveis e o conceito de "equilíbrio com desemprego".

De acordo com Vercelli (1991:12) existem dois conceitos de equilíbrio usados na teoria econômica, a saber:

- Conceito sintático de equilíbrio, segundo o qual o mesmo é definido como a solução de um sistema de equações. Nesse caso, o equilíbrio nada mais é do que o conjunto de valores para as variáveis endógenas de um sistema de equações que torna o mesmo logicamente consistente.

- Conceito dinâmico de equilíbrio, segundo o qual o equilíbrio é visto como uma posição de repouso do sistema econômico, isto é, uma situação na qual o sistema está desprovido de qualquer tipo de dinâmica endógena. O equilíbrio, nesse caso, é idêntico ao conceito de steadystate.

O conceito de equilíbrio com desemprego só pode ser entendido se o equilíbrio for definido no sentido dinâmico, não com base no sentido estático. Isso porque uma situação na qual existe desemprego involuntário da força de trabalho consiste numa situação em que o número de pessoas dispostas a trabalhar ao salário de mercado supera o número de empregos disponíveis a esse mesmo salário. Do ponto de vista estático, isso é o mesmo que dizer que a condição de "esvaziamento" (market clearing) do mercado de trabalho não é satisfeita, de forma que o sistema de equações não apresenta uma "solução."

Os intérpretes de Keynes são unânimes na constatação de que o conceito de equilíbrio usado na TG é o dinâmico, não o estático. Sobre essa questão, Davidson (1998:23) afirma que:

The equilibrium concept, however, was brought into economics by Marshall who borrowed it from physics where equilibrium means a balancing of endogenous forces so that the body under study is 'at rest'. Keynes, a student of Marshall, insisted that in an involuntary unemployment equilibrium there are no endogenous free markets forces that would automatically alter the existing balance of market forces to chance the unemployment equilibrium position even if prices are perfectly flexible. The concept of clearing is not necessary for a bodyat-rest economic equilibrium.

Dessa forma, uma posição de equilíbrio com desemprego na qual os salários nominais são flexíveis deve ser entendida como uma situação 
na qual a redução dos salários nominais resultante do desemprego da força de trabalho não gera nenhuma tendência à alteração do nível de produção e emprego da economia. Isso só vai acontecer, contudo, se $a$ demanda realizada for insensível às variações da taxa nominal de salários. Essa condição exige que os efeitos favoráveis e desfavoráveis da deflação dos salários nominais sobre a demanda realizada listados por Keynes no capítulo 19 da TG se cancelem mutuamente. Embora essa possibilidade não possa ser a priori descartada, claramente trata-se de uma "feliz coincidência" e, portanto, de um resultado cuja ocorrência não pode ser, em geral, esperada. Via de regra, podemos esperar que os efeitos desfavoráveis prevaleçam sobre os efeitos favoráveis (Tobin 1997:20), de tal maneira que a redução dos salários nominais irá resultar numa contração do nível de produção e de emprego.

Desse razoado se segue que se economia estiver operando com desemprego involuntário da força de trabalho, então a redução resultante dos salários nominais - no caso em que os mesmos são flexíveis - irá produzir um ciclo vicioso de queda da demanda realizada-queda do nível de produção e de emprego-nova queda dos salários nominais. Esse processo não conduz a economia a posição de repouso alguma. Pelo contrário, trata-se de um processo dinâmico explosivo no qual o nível de produção e de emprego tende assintoticamente a zero! Daqui se segue que a flexibilidade de salários nominais irá resultar, em geral, num processo de desequilíbrio caracterizado por desemprego crescente (e involuntário) da força de trabalho. Sendo assim, o aspecto essencial da teoria do emprego de Keynes não é o equilíbrio com desemprego, mas o desemprego como o resultado de uma economia que se move ao longo do tempo em estado de permanente desequilíbrio. Essa interpretação já havia sido antecipada por Patinkin (1948:280):

\footnotetext{
The fundamental issue raised by Keynesian economics is the stability of the dynamic system: its ability to return automatically to a full employment equilibrium with in a reasonable time if it is subjected to the customary shocks and disturbances of the peacetime economy. In other words, what Keynesian economics claims is that the economic system may be in a position of underemployment disequilibrium (in the sense that wages, prices, and the amount of unemployment are continuously changing over time) for long, or even indefinite, periods of time.
}

É neste ponto que encontramos o elemento fundamental de discórdia entre Davidson e Tobin. Para Davidson (1998:22), Keynes teria construído uma teoria do equilíbrio com desemprego de longo-período. Nas suas palavras: 
Keynes anchored his argument for the possibility of a stable long-period underemployment equilibrium in his essential properties of money chapter (...). Only by ignoring this chapter can Tobin assert that "all Keynesian macroeconomics really requires is that product prices and money wages are not perfectly flexible."

Essa interpretação de Davidson está certamente apoiada na seguinte afirmação que Keynes (1936:48) faz na sua TG:

\begin{abstract}
If we suppose a state of expectations to continue for a sufficient length of time for the effect on employment to have worked itself out so completely that there is (...) no piece of employment going on which would not have taken place if the new state of long-term expectation had always existed, the steady level of employment thus attained may be called the long-period employment corresponding to that state of expectations. It follow that, although expectations may change so frequently that the actual level of employment has never had time to reach the long-period employment corresponding to the existing state of expectations, nevertheless, every state of expectations has its definite corresponding level of long-period employment.
\end{abstract}

Na citação acima de Keynes fica claro que o conceito de "equilíbrio de longo-período" refere-se a uma situação na qual o nível de emprego se ajustou perfeitamente ao estado de expectativas de longo-prazo, o qual determina as decisões de investimento dos empresários. Esse ajuste perfeito do emprego ao estado de expectativas é que daria o caráter de "longo-período" ao equilíbrio obtido no modelo de Keynes. ${ }^{15}$

O conceito de "equilíbrio" ou "posição" de longo-período tem uma longa tradição na teoria econômica, remontando aos economistas clássicos e Marx (cf. Milgate 1982:12). Para esses autores, uma economia capitalista estaria numa posição de longo-período se a taxa de lucro fosse uniforme em todos os setores de atividade. Para tanto, seria necessário que o tamanho e a composição do estoque de capital fossem adequados ao tamanho e à composição da demanda pelos produtos obtidos a partir do mesmo (Milgate 1982:12). Daqui se segue que uma posição (de equilíbrio) de longo-período exige não apenas o ajuste do emprego ao estado de expectativas, como também o ajuste do tamanho e da composição da capacidade produtiva ao tamanho e à composição da demanda realizada. No entanto, a teoria do emprego de Keynes supõe que o estoque de capital, o tamanho e a qualidade da força de trabalho, bem como a tecnologia, são dados (cf. Keynes 1936:23). Sendo assim, se o conceito de "equilíbrio de longo-período" for empregado de forma consistente com a definição usual desse termo, não há nenhuma base 
objetiva para se afirmar que a teoria do emprego de Keynes define uma posição de "equilíbrio de longo-período" com desemprego involuntário da força de trabalho.

Além disso, uma das hipóteses subjacentes ao suposto equilíbrio de longo-período com desemprego é que a taxa nominal de salários é um dado, conforme podemos constatar na citação abaixo de Rogers (1997:338): "It is clear from the above discussion that the existence of a long-period equilibrium in statical economics rests on the assumption of given longterm expectations, E, and money-wages, $\mathrm{W}$, in particular."

Dessa forma, podemos constatar que a interpretação de Davidson da teoria do emprego de Keynes, apoiada também por Colin Rogers, além de estar baseada num conceito equivocado de "equilíbrio de longo-período", não lida com a questão teórica fundamental apresentada por Tobin, a saber: a incompatibilidade entre os efeitos dinâmicos da flexibilidade da taxa nominal de salários e o conceito de equilíbrio (empregado por Keynes na TG) como uma posição de repouso do sistema econômico. Sendo assim, o veredicto final sobre o debate em consideração não poderia ser outro do que o apresentado por Tobin (1975:195):

\begin{abstract}
Keynes's General Theory attempted to prove the existence of equilibrium with involuntary unemployment, and this pretension touched a long theoretical controversy. (...) The predominant verdict of history is that, as a matter of pure theory, Keynes failed to prove his case.
\end{abstract}

A flexibilidade da taxa nominal de salários obriga o abandono do conceito de equilíbrio como expediente metodológico para a apresentação das idéias de Keynes. Isso não implica na aceitação de que a rigidez de salários nominais é a causa do desemprego ou que não existe papel para a intervenção governamental no longo-prazo. A teoria do emprego de Keynes é totalmente compatível com a hipótese de salários nominais flexíveis desde que a mesma seja apresentada em termos dinâmicos, ao invés de estáticos. ${ }^{16}$

16 Uma possível explicação para a insistência dos pós-keynesianos em apresentar a teoria do emprego de Keynes em termos de um referencial de equilíbrio é que o desequilíbrio é visto por esses autores como um fenômeno transitório. Nas palavras de Rogers: "Keynes, it is usually asserted, restricted his theoretical analysis to short-period equilibrium or disequilibrium adjustments. (...) On this view unemployment is a temporary disequilibrium phenomenon" (1997:325). Essa interpretação do desemprego de desequilíbrio é, ao nosso ver, incorreta, uma vez que o processo dinâmico descrito por Keynes no capítulo 19 da TG é essencialmente explosivo e, portanto, divergente com relação à posição de equilíbrio com pleno-emprego. 


\section{Conclusão}

Ao longo do presente artigo, argumentamos que a teoria do emprego de Keynes não está baseada na hipótese de reversão de velocidade relativa de ajuste entre preços e quantidade, tal como afirmado por Leijonhufvud, Friedman e Tobin. O modelo de Keynes apresenta uma economia que opera em condições competitivas e onde os preços variam no interior do período de produção para assegurar o ajuste entre o nível de produção escolhido pelas firmas no início do período e a demanda realizada pelos seus produtos. Nesse contexto, uma variação não-antecipada da demanda realizada dará ensejo a uma variação do nível de preços relativamente às expectativas das firmas sobre essa variável, causando assim lucros ou prejuísos extraordinários. Como resposta aos mesmos, as firmas irão alterar o nível de produção e de emprego no próximo período.

Ao longo de um dado período de produção, os salários nominais são fixos, uma vez que os mesmos resultaram de uma negociação entre firmas e trabalhadores no início do período. Os salários nominais podem, no entanto, variar ao longo de uma seqüência de períodos de produção, de forma que a teoria do emprego de Keynes não pressupõe salários nominais fixos. Com efeito, Keynes dedica o capítulo 19 da TG à análise dos efeitos da flexibilidade (em tempo real) dos salários nominais sobre a demanda agregada. Com base nos argumentos expostos por Keynes nesse capítulo, podemos concluir que a relação entre a taxa nominal de salários e o nível de demanda realizada pode ser não-negativa, de tal maneira que a flexibilidade de salários pode terminar por amplificar, ao invés de reduzir, o desemprego da força de trabalho. Sendo assim, a existência de desemprego involuntário não pressupõe a rigidez da taxa nominal de salários.

Isso não significa, contudo, que Keynes tenha demonstrado a existência de uma posição de equilíbrio com desemprego num contexto em que os salários nominais são flexíveis. De fato, os argumentos apresentados por Keynes no capítulo 19 da TG apontam para a idéia de que a flexibilidade da taxa nominal de salários irá gerar um ciclo vicioso de queda da demanda realizada-aumento do desemprego-queda dos salários nominais-nova queda da demanda realizada. Esse argumento é per se incompatível com o conceito de equilíbrio no sentido usado por Keynes na sua TG, ou seja, o equilíbrio como uma posição de repouso do sistema econômico. Daqui se segue que a essência da teoria do emprego de Keynes deve ser apresentada em termos dinâmicos, ao invés de estáticos, e que o conceito de "equilíbrio com desemprego" deve ser substituído pela noção de "desemprego de desequilíbrio", tal como sugerido por Patinkin e Tobin. 


\section{Referências}

AMADEO, E. J. (1989). Keynes's principle of effective demand. Aldershot: Edward Elgar.

(1992). "A Revolução Keynesiana e a análise do multiplicador" In AMADO, A. M. \& FALCÃO, M. L. (orgs). Moeda e produção: teorias comparadas. Brasília: Editora da UNB.

ASIMAKOPULOS, A. (1991). Keynes's General Theory and accumulation. Cambridge: Cambridge University Press.

CARVALHO, F. C. (1992). Mr Keynes and the post Keynesians: principles of macroeconomics for a monetary production economy. Aldershot: Edward Elgar.

CHICK, V. (1993). Macroeconomia pós-Keynes: um reexame da Teoria Geral. Rio de Janeiro: Forense Universitária.

DAVIDSON, P. (1997). "Did Keynes reverse the Marshallian speeds of adjustment? Using Harcourt's Method to resolve theoretical controversies and to gain insight into real world." In ARESTIS, P. \& PALMA, G. \& SAYWER, M. (orgs). Capital controversy, post Keynesian economics and the history of economic thought: essays in honour of Geoff Harcourt. vol. 1. Londres: Routledge.

(1998). "Setting the record straight" In ROTHEIM, R. (org.). New Keynesian economics-post Keynesian alternatives. Londres: Routledge.

DUTT, A. K. (1992). "Expectations and equilibrium: implications for Keynes, the neo-Ricardian Keynesians and the post Keynesians." Journal of Post Keynesian Economics 14(2).

KEYNES, J. M. (1936). The general theory of employment, interest and money. Londres: Macmillan Press.

LEIJONHUFVUD, A. (1968). On Keynesian economics and the economics of Keynes. Nova Iorque: Oxford University Press.

(1981). Information and coordination: essays in macroeconomic theory. Nova Iorque: Oxford University Press.

MODIGLIANI, F. (1944). "Liquidity preference and the theory of interest and money." Econometrica 12.

MILGATE, M. (1982). Capital and employment: a study of Keynes's economics. Nova Iorque: Academic Press.

PATINKIN, D. (1948). "Price flexibility and full employment." American Economic Review 38.

ROGERS, C. (1997). "Existence of a monetary long-period equilibrium" In HARCOURT, G. \& RIACH, P. (orgs). A second edition of the General Theory. vol. 1. Londres: Routledge.

SOLOW, R. \& HAHN, F. (1986). A critical essay on macroeconomic theory. Cambridge: MIT Press.

TOBIN, J. (1975). "Keynesian models of recession and depression." American Economic Review 75. 
(1993). "Price flexibility and output stability: an old Keynesian view." Journal of Economic Perspectives 7(1).

(1997). "An overview of the General Theory" In HARCOURT, G. \& RIACH, P. (orgs). A second edition of the General Theory. vol. 2. Londres: Routledge.

VERCELLI, A. (1991). Methodological foundations of macroeconomics: Keynes and Lucas. Cambridge: Cambridge University Press.

Artigo recebido em 23/05/2006.

Aceite em 22/06/2006. 